International Journal of Biology, Pharmacy and Allied Seiences (IJBPAS)

'A Bridge Betuen caboratory and Q Qnder'

WwW.ibpas.com

\title{
A REVIEW: IMPACT OF GARLIC ON HUMAN HEALTH
}

\section{DEVI A ${ }^{* 1}$, CHAURASIA H ${ }^{2}$, CHANDEL $S^{3}$, KAUSHIK $S^{4}$ AND BHATT $B^{5}$}

1*: Research Scholar, Adarsh Vijendra Institute of Pharmaceutical Sciences, Shobhit University Gangoh, Saharanpur, (Uttar Pradesh)

2: Associate Professor, Adarsh Vijendra Institute of Pharmaceutical Sciences, Shobhit University Gangoh, Saharanpur, (Uttar Pradesh)

3: Department of Microbiology, Career Point University, Hamirpur (Himachal Pradesh)

4: Research Scholar, Adarsh Vijendra Institute of Pharmaceutical Sciences, Shobhit University Gangoh, Saharanpur, (Uttar Pradesh)

5: Research Scholar, Adarsh Vijendra Institute of Pharmaceutical Sciences, Shobhit University Gangoh, Saharanpur, (Uttar Pradesh)

*Corresponding Author: Anjana Devi: E Mail: anjana.sapna@gmail.com

Received $14^{\text {th }}$ May 2020; Revised $11^{\text {th }}$ June 2020; Accepted $16^{\text {th }}$ July 2020; Available online $1^{\text {st }}$ March 2021 https://doi.org/10.31032/IJBPAS/2021/10.3.5404

ABSTRACT

The medicinal plants are worldwide for treatment of arthritis. The medicinal plants throughout the world used by various tribes groups for treatment of arthritis. India has a biggest rich of medicinal plants in the production of raw material, crude drugs and in the formulations of pharmaceuticals and cosmetics etc. Plants play a vital role for the existence of life on the earth. People play important role in using local plants as traditional herbal therapies and folk remedies. The wild species are spiritual, religious and medicinal plant, native of North-West Himalayan region of India and spread throughout South East Asia. The plant as a medicine is used in different system of medicine such as Ayurveda, Allopathy, Unani and Homeopathy. The people use the plants and products to meet their daily or basic needs such as food, clothes, fuel, shelter, fiber and mainly medicines for treating or curing primary health problems. The local people or rural people of this region have good faith in the traditional knowledge about the herbal potential of local plants for alleviating different 
types of health problems that are prevailing in this region. The extraction of secondary metabolites from these plants will be performed through standard operating procedures. Organically these raw materials will be intermingled to make immunity enhancer paste. Fruit and stems of these plants are used for various therapeutic purposes, such as for treatment of joint pain and bodyache.

\section{Keywords: -Allium sativum, Herbal medicine, Traditional medicine, Anti Arthritic} effect, Anti Microbial effects

\section{INTRODUCTION}

In past years, Garlic has been used in various cultures to combat infectious disease. It is also used as food flavoring as well as traditional medicines. The garlic plant's bulb is most commonly used part of the plant. The single clove types, garlic bulbs divided into fleshy sections called cloves. Garlic cloves are used for consumption (raw or cooked) or for medicinal purposes like alzheimer's disease, anti-arthritic, antimicrobial, acnereducers, cold and flu, antifungal, reduces blood pressure etc [1].

Rheumatoid arthritis is a chronic, systemic autoimmune disease that involves inflammation in the membrane lining of the joints and often affects internal organs of human body. Many in vitro studies also show the antibacterial effect of garlic extract against human dental plaque micro biota. Many researchers show the synergistic effect of garlic with various antibiotics. Similarly, the synergistic effect of garlic extract with between ciprofloxacin has been shown, but not effect shown with ampicillin. The cloves of garlic are extracted with $95 \%$ ethanol. The antibacterial activity against multi-drug clinical pathogens, can be used for prevention and treatment of drug resistant during microbial diseases [2]. Garlic (Allium sativum L.) is a common spice with many health benefits, mainly due to its diverse bioactive compounds, such as organic sulfides, saponins, phenolic compounds, and polysaccharides. Garlic is most commonly consumed. During recent years, many researchers have demonstrated the significant biological functions of garlic, including anticancer, cardiovascular protective, anti-inflammatory, immunemodulatory, anti-diabetic, anti-obesity, antioxidant and antibacterial properties. Therefore we are focusing on significant property ofgarlic in human health. For that we studied high-quality researcher article from the lastfive years and reviewed the main bioactive compounds as well as biological functions of garlic, with special attention paid to the relevant mechanisms 
of actions. This review paper will attract more interest in garlic and provide efficient scientific evidence for the better utilization of garlic in human health and disease controlling [3].

Table 1: Scientific classifications of Allium sativum

\begin{tabular}{|c|c|}
\hline Kingdom: & Plantae \\
\hline Clade: & Tracheophytes \\
\hline Clade: & Angiosperms \\
\hline Clade: & Monocots \\
\hline Order: & Asparagales \\
\hline Family: & Amaryllidaceae \\
\hline Subfamily: & Allioideae \\
\hline Genus: & Allium \\
\hline Species: & A. sativum \\
\hline
\end{tabular}

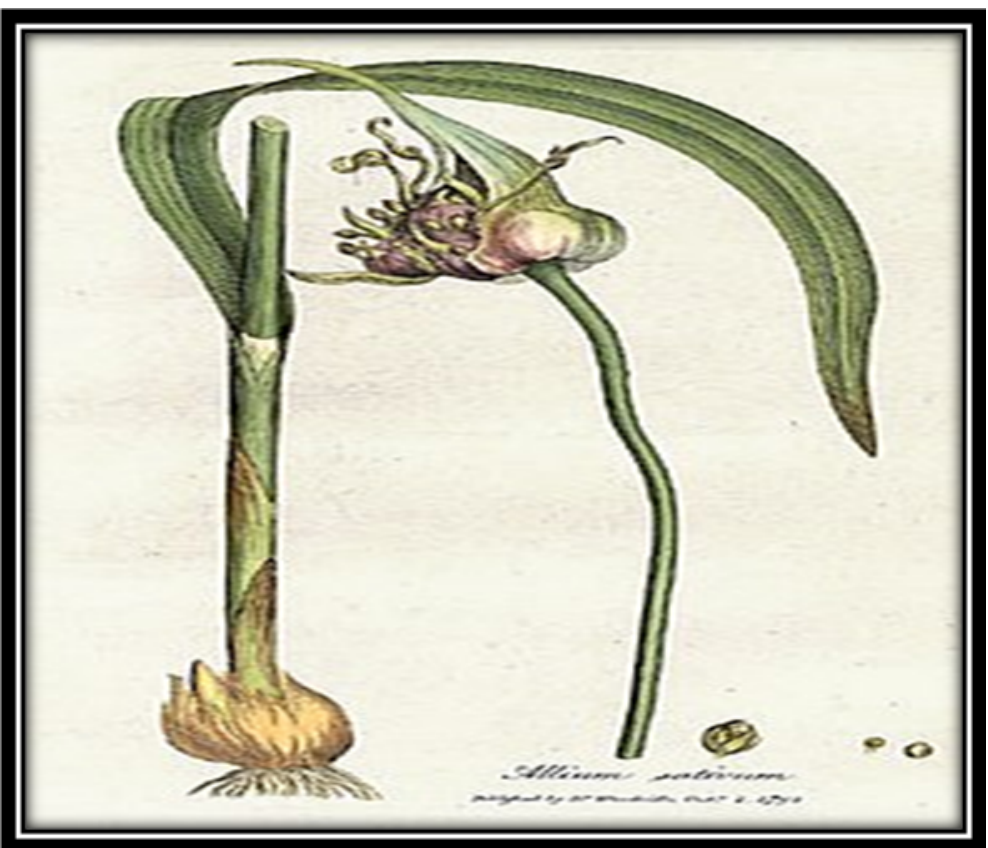

Figure 1: This figure showing the plant of Allium sativum

\subsection{Origin and distribution:}

Garlic is among the oldest known horticultural crops. In the Ancienttime, Egyptian and Indian cultures mentioned to garlic 5000 years ago and there is clear historical evidence for its use [4]. The garlic propagated wildly over a much larger region. This region where garlic has grown with in the wild is referred to "center of origin" this is often the geographic region. Where the crop originated and therefore theonly place where it flourished. Although we sometimes, hear about "wild garlic" elsewhere in the world, this is the only region where true garlic grows in the wild without the assistance of human propagation. There are other plants locally referred to as "wild garlic", but these are invariably other species of the garlic genus, not garlic itself [5] For example, Allium vineale is a wild family member of garlic that occurs in North America, commonly 
called "wild garlic" (USDA, 2006). That is why those who have required finding new genetic variation in garlic have collected wild garlic in Central Asia. It was not until garlic was cultivated in southern Europe within the last 1000 years that the distinction between hardneck and softneck was routinely noted [6]. Until more ancient writings which describe garlic are found, or old, well-preserved samples are unearthed, we can only speculate about the early types of garlic grown. The cost of cultivation of garlic in Kullu valley was estimated at Rs. 617 per quintal, excluding family labour and Rs. 1027 per quintal, including family labour [7]. Garlic variety Agrifound parvati $(27.50 \mathrm{t} / \mathrm{ha})$ gave the highest yield followed by GHC-1(25.20 t/ha).Among crop sequences tested at Kangra, maize(grain)-frenchbean-garlic and maize +cucumber-pea giving net returns of Rs.2,37,340and Rs.1,77,440, respectively have potential. Higher productivity of garlic [8].

\section{PHARMACOLOGICAL}

\section{PROPERTIES}

\subsection{Anti -Arthritic Activity:}

The method of scrutining the information for this critical review article includes those plants: (i) Which are native to India and other countries like America, Africa or Europe, (ii) utilized in ancient systems and in numerous polyherbal preparations, (iii) with reported anti-arthritic activity, (iv) appropriate dosage, (v) mechanism of action, (vi) safety profile, and (vii) models used [9]. The extracts of medicinal plants used traditionally in acute rheumatic attacks, chronic analgesia, and chronic rheumatism have been considered as antiarthritic agents. The poly herbal formulations for Arthritis Analgesics are useful in decreasing pain and inflammation in either acute or chronic RA patients. Although the treatment of RA is available but due to potential adverse effects or irreversible organ damage the new approaches are developed for maintaining the balance between these potential risks and acknowledged benefits [10]. A Unani polyherbal formulation was evaluated for its antiarthritic activity in rats [11-13]. The anti-arthritic activity of formulation was studied using the collagen induced arthritis model in wistar rats and it was revealed that at the doses of 100 and $300 \mathrm{mg} / \mathrm{kg}$.

\subsection{Antioxidant Activity:}

Antioxidants play vital role in maintaining balance between free radical produced by metabolism or derived from environmental conditions and antioxidant system of the body. Due to deficiency of antioxidants agents in human body leads to enhance oxidative damage. Antioxidants are commonly observed in medicinal plants are effective and safe due to their harmless 
nature and have been reported to have multiple biological effects. Among medicinal plants, the plants of genus Allium are known to very useful for their healing potentials. Superoxide is dramatically increased during stressful condition. This superoxide is one of the main reactive oxygen species in the cell which are chemically reactive molecules containing oxygen also increased [14-16]. In this study allicin has brought about substantial increase in these reactive oxygen species including superoxidase dismutase, catalase and glutathione peroxidase and also DPPH enzyme which was statistically significant compared to control as well as comparable to standard drug Piroxicam. Therefore this study clearly demonstrates that the active constituent of Allium sativum which is Allicin has displayed significant antiarthritic activity in vivo in albino rats. This active component also showed significant antioxidant activity in vitro via the enzymes superoxidase dismutase, catalase and glutathione peroxidase and also via DPPH enzyme [17].

\subsection{Anti-Inflammatory Activity:}

Garlic and active compounds have also been shown to display anti-inflammatory activity. In this study, the ethyl linoleate available in garlic reduced the production of nitric oxide (NO) and prostaglandin E-2 by down-regulating the expression of inducible NO synthase (iNOS) and cyclooxygenase-2 (COX2) in lipopolysaccharide-stimulated. The treatment of AGE reduced the level of tumor necrosisfactor- (TNF-) and the IL-1 receptor-associated kinase 4 and enhanced the activity of the adenosine monophosphate-activated protein kinase (AMPK) in the liver. Moreover, allicin could be usedas a potential complementary treatment against the inflammatory response induced by schistosome infection in mice. Furthermore, garlic supplements alleviated osteoarthritis in obese oroverweight patients by reducing resistin. Collectively, in both in vitro and in vivo experiments, garlic could inhibit inflammation mainly by inhibiting inflammatory mediators, such as NO, TNF, and IL-1. Garlic has great potential to treat inflammatory diseases, such as arthritis, in humans, because of its low or absent toxicity [18].

\subsection{Antimicrobial Activity:}

Garlic has a extensive range of antibacterial and antifungal potential. It was shown in studies that garlic oil inhibited the fungus Penicillium funiculosum, possibly by penetrating into cells and organelles or destroying the cell structure and inducing theleakage of cytoplasm and macromolecules [19-20]. Additionally, garlic oil was found to disrupt the normal 
metabolism of Candida albicans, which is associated with the induction of key genes involved inoxidative phosphorylation, the cell cycle, and protein processing in the endoplasmic reticulum. Furthermore, in a clinical trial, the treatment of raw garlic inhibited Helicobacter pylori in the stomach of patients with $H$. pylori infection. In a word, the antibacterial eects of garlic are related to its varieties and processing methods. Garlic oil was demonstrated to be the main antibacterial ingredient that destroys the structure and the metabolic process of bacterial cells [21].

\subsection{Immunomodulatory Activity:}

Garlic contains various bioactive compounds that are beneficially for the immune system. Compared with black garlic, polysaccharides in fresh garlic exhibit a more potent activity in immunomodulation. This is probably due to the degradation of fructan constituents during processing. The combination of garlic oil and levamisole can significantly balance the T-helper response in the Wistar rats. Furthermore, selenylation modification of garlic polysaccharides significantly improves its immune-enhancing activity, and selenizing garlic polysaccharides promote lymphocyte proliferation, enhance interferon-and IL-2, and increases the serum antibody titer in 14-day-old chickens. Moreover, the ingestion of AGE was observed to reduce the occurrence and severity ofthe cold and flu and improve the immune system functions in humans. Overall, it can be concluded that polysaccharides appear to be the main immune-modulating components in garlic [22-24].

\subsection{Antihypertensive Activity:}

Garlic may be reduce oxidative stress and increase the production of Nitrous Oxide and hydrogen sulfide (H2S). And it may be inhibiting the angiotensin converting enzyme, lowering hypertension. A study showed that AGE could stimulate the production of NO, leading to endothelialdependent vasodilationin the isolated rat aortic rings. Moreover, 1-arginine in AGE was crucial in the NOS-mediated NO production. In addition, S-1-propylenecysteine was shown to be the key antihypertensive compound in the AGE. S1-propylenecysteine improves peripheral blood circulation. It may be reduce the systolic blood pressure in spontaneous hypertension rats, without affect the systolic blood pressure of control rats. In vivo study, the nitrites in the fermented garlic extract (FGE) could be converted into NO by Bacillus subtilis. Further, NO reduced the systolic bloodpressure in spontaneous hypertension rats through the soluble guanylyl cyclase (sGC)-cyclic guanosinemonophosphate (cGMP)-protein 
kinases G (PKG) pathway [25-26]. Fermented garlic extract was shown to alleviate pulmonary hypertension by reducing the expression of vascular endothelial cell adhesion molecule-1. The combination of garlic and its bioactive compound alliin and captopril, improved the activity ofcaptopril on inhibiting angiotensin-converting enzyme (ACE) and hypertension in rats.

\subsection{Anti-hyperlipidemicActivity:}

Garlic can lower blood lipids in animals and people. A study exhibited that high temperature and high pressure handling could remove the pungency of garlic and this garlic effectively reduced the levels of total cholesterol, low-density lipoprotein cholesterol, and triglyceride in highcholesterol diet. Another study found that adding $1.5 \%$ black garlic extract in high-fat diet for male Sqrague-Dawley rats could significantly modulate the metabolism of lipids and cholesterol and decrease the total levels of blood lipids, triglyceride, and cholesterol, which could be due to the reduction of the mRNA expression of sterol regulatory element binding protein-1c. In a cross sectional study, the intake of garlic (300 mg/day, 8 weeks) was shown to reduce the levels of cholesterol and lowdensity lipoprotein and elevate the level of high-density lipoprotein, but garlic had no effect on the level of triglycerides in patients with diabetic dyslipidemia [27]. The supplement of garlic for 13 weeks was observed toreduce the actions of myeloperoxidase and lipid hydroperoxide in serum. The mechanisms of the antihypertensive properties of garlic extract via increasing theproduction of nitric oxide (NO) in vascular smooth muscle cells. Moreover, the nitrite in the fermented garlic extract (FGE) could be converted into NO in vivo by Bacillus subtilis. NO and atrial natriuretic peptide (ANP) activated particulate guanylyl cyclase (pGC) and soluble guanylyl cyclase (sGC), thus catalyzing the transform of guanosine triphosphate (GTP) tocyclic guanosine monophosphate (cGMP) [28].

\subsection{Anticancer Activity:}

Cancer is a leading cause of death in the whole world. The numerous natural medicinal products such as berries, vegetables, tomatoes, garlic have constituted anticancer properties. Various studies have also revealed that garlic and its constituents can protect against diverse cancers, such as colorectal, lung, gastric, and bladder cancers. Additionally, the garlic and lemon aqueous extract were revealed to activate the immune system against implanted breast cancerin mice by increasing interferon- IL-2, and IL-4 level. Garlic and its active components are responsible to prevent and manage different 
cancers. These anticancer mechanisms include the regulation of carcinogen metabolism, inhibition of cell growth and proliferation, initiation of apoptosis, suppression of angiogenesis, and inhibition of invasion and migration. Garlic can also reduces the negative effect of anticancer therapies [29-30].

\subsection{Hepatoprotective Activity:}

Various studies have exposed that natural products with garlic had hepato-protective effects. In an in vitro study, the black garlic extract decreased the damage of tert-butyl hydroperoxide in rat clone-9 hepatocytes by inhibiting apoptosis, lipid peroxidation, oxidative stress, and inflammation. In another study, garlic attenuated liver damage inducedby alloxan in rats and improved the biochemical plasma factors of hepatic functions, such as urea, creatinine, aspartate transaminase, and alanine transaminase [31]. Moreover, single clove garlic had a stronger protective effect. Moreover, garlic oil was shown to enhance the activities of hepatic antioxidant enzymes, block metabolic activation of 1 , 3-dichloro-2-propanol, and reduce apoptosis in the liver, indicating a protective effect against liver injury in rats. Garlic essential oil was found to attenuate nonalcoholic fatty liver disease, which was induced by a high-fat diet in rats. The release of pro-inflammatory cytokines in the liver and increased antioxidant activity by inhibiting the expression of cytochrome .Compared with unfermented garlic extract, the fermented garlic extract by Lactobacillus plantarum was able to more eectively reduce liver lipid levels and ameliorate hepatic steatosisin mice. Furthermore, considered as a potential treatment for mild hepatic dysfunction. In a double-blind, randomized, placebocontrolled study, 36 adults with mildly high level of serum gamamyl glutamyl transpeptiase (GGT) received. However, an animal study showed that an overdose of garlic has negative morphological effect onthe liver. After 30 days of injecting fresh garlic extract at $500 \mathrm{mg}$ and $1000 \mathrm{mg} / \mathrm{kg}$. Generally speaking, garlic can effectively alleviate acute or chronic liver injury, but the side effect of excessive consumption of garlic also need to be considered [32-34]. It is necessary to evaluate the safe dose and duration of garlic usage in humans.

\subsection{Anti-Diabetic Activity:}

Garlic has been found to decrease pancreatic cell injury, oxidative stress, and pathological changesin streptomycininduced type 1 diabetic rats. Garlic had a protective effect against diabetic etinopathy in diabetic rats. The weight, blood glucose and morphological changes of retinal tissue treated with garlic improved after 8 weeks of gastric gavage of raw garlic extract in 
rats. Moreover, AGE had a dose-dependent anti-diabetic eect on streptomycin-induced diabetic rats [35]. Furthermore, a metaanalysis was performed on 768 patients with type 2 diabetes mellitus in nine randomized controlled trials, and the result showed that garlic supplements significantly reduced fructosamine and glycosylated hemoglobin. This study demonstrated that garlic supplements were effective in the management of type 2 diabetes mellitus. Thus, garlic and its bioactive components might be effective agents to help treat diabetes and diabetic complications [36].

\subsection{Anti-Obesity Activity:}

Garlic oil has anti-obesity properties and has been shown to counteract the influence of a high-fat diet on the weight of body and adipose tissue in hyperlipidemia rats. LAFGE also reduced their epididymal, retroperitoneal, and mesenteric adipose tissue mass. The possible mechanism of action was that LAFGE inhibited lipogenesis by down-regulating the mRNA and protein expression of PPAR-C/EBP, and lipogenic proteins. Moreover, the methanolic extract of black garlic was found to reduce the weight of rats fed with a high-fat diet. This treatment regulated lipid metabolism by up-regulating the expression of AMPK, forkhead box protein O1, perilipin, and adiponectin in the adipose tissue of the rats and downregulating the cluster of dierentiation 36 (CD36), plasminogen activator inhibitor 1, resistin, and TNF [37-40]. The studies demonstrate that fermented garlic products have certain positive effecton obesity by inhibiting lipogenesis and regulating lipid metabolism.

\section{CONCLUSION}

Garlic is a generally consumed spice with a distinguishing odor. Many bioactive compounds are present in garlic, such as organic sulfides, saponins, phenolic compounds, and polysaccharides. The organic sulfides, such as allicin, alliin, diallyl sulfide, diallyl disulfide, diallyl trisulfide, ajoene, and S-allyl-cysteine, are major bioactive components in garlic. Garlic and its major bioactive components exhibited many biological functions including digestive system protective antioxidant, anti-inflammatory, immunemodulatory, cardio-vascular protective, anticancer, hepato-protective, anti-diabetic, anti-obesity, neuro-protective, renal protective, antibacterial, and antifungal activities. Garlic used as medicinal property having non-toxic or low toxicity. Therefore, garlic is encouraging as functional foods for the prevention and treatment of different diseases. Hence, more biological functions of garlic should be assessed and the relative compounds of 
garlic need to be separatedand identified. Therefore many researchers should be conducted to deeply illustrate garlic's mechanisms of action. Furthermore, more clinic trials should be carried out to confirm the health benefits of garlic on humans, and special attention should be paid to the side effect/safety of garlic.

\section{REFERENCES}

[1] Ademiluyi AO, Oboh G, Owoloye TR, Agbebi OJ. Modulatory effects of dietary inclusion of garlic (Allium sativum) on gentamycininduced hepatotoxicity and oxidative stress in rats. Asian Pac J Trop Biomed. 2013; 3:470-475

[2] Adetumbi M, Javor GT, Lau BH. Allium sativum (garlic) inhibits lipid synthesis by Candida albicans. Antimicrob. Agents Chemother. 1986; 30: 499-501.

[3] Allison GL, Lowe GM, Rahman K. Aged garlic extract inhibits platelet activation by increasing intracellular cAMP and reducing the interaction of GPIIb/IIIa receptor with fibrinogen. Life Sci. 2012; 91: $1275-1280$

[4] Amagase H, Milner JA. Impact of various sources of garlic and their constituents on 7, 12- dimethylbenz [a] anthracene binding to mammary cell DNA. Carcinogenesis. 1993; 14:1627-1631.

[5] Ashraf R, Aamir K, Shaikh AR, Ahmed T. Effects of garlic on dyslipidemia in patients with type 2 diabetes mellitus. J Ayub Med Coll Abbottabad. 2005; 17:60-64.

[6] Auer W, Eiber A, Hertkorn E, Hoehfeld E, Koehrle U, Lorenz A, Mader F, Merx W, Otto G, SchmidOtto B, et al. Hypertension and hyperlipidaemia: garlic helps in mild cases. $\mathrm{Br} \mathrm{J}$ Clin Pract Suppl. 1990; 69: 3-6.

[7] Bakhshi M, Taheri JB, Shabestari SB, Tanik A, Pahlevan R. Comparison of therapeutic effect of aqueous extract of garlic and nystatin mouthwash in denture stomatitis. Gerodontology. 2012; 29: e680-684.

[8] Banejee SK, Maulik SK. Effect of garlic on cardiovascular disorders: a review. Nutr J. 2002; 1:4

[9] Bordia A, Verma SK, Srivastava KC. Effect of garlic (Allium sativum) on blood lipids, blood sugar, fibrinogen and fibrinolytic activity in patients with coronary artery disease. Prostaglandins Leukot Essent Fatty Acids. 1989; $58: 257-63$. 
[10] Antman EM, Bennett JS, Daugherty A, et al. Use of nonsteroidal anti-inflammatory drugs. An update for clinicians. A scientific statement from the American Heart Association. Circulation 2007; 115: 1634-42.

[11] Singh, Y udhvir and Chand, Ramesh 2003. Performance studies of some garlic clones.

[12] Himachal Journal ofAgricultural Research 29 (1 \& 2): 35-42.

[13] Matysiak, M.; Binduga, U.E.; Gminski, J. Characterization of active compounds of different garlic (Allium sativum L.) cultivars. Pol. J. Food Nutr. Sci. 2018, 68, 73-81.

[14] Boonpeng, S.; Siripongvutikorn, S.; Sae-Wong, C.; Sutthirak, P. The antioxidant and anti-cadmium toxicity properties of garlic extracts. Food Sci. Nutr. 2014, 2, 792-801.

[15] Lee, D.Y.; Li, H.; Lim, H.J.; Lee, H.J.; Jeon, R.; Ryu, J.-H. Antiinflammatory activity of sulfurcontainingcompounds from garlic. J. Med. Food. 2012, 15, 992-999.

[16] Aqueous garlic extracts trigger antioxidants. Front. Plant Sci. 2016, 7, 1235. [CrossRef]
[PubMed] Foods 2019, 8, 24624 of 31

[17] Percival, S.S. Aged garlic extract modifies human immunity. J. Nutr. 2016, 146, 433S-436S.

[18] Lee, H.S.; Lim, W.C.; Lee, S.J.; Lee, S.H.; Lee, J.H.; Cho, H.Y. Antiobesity ect of garlic extract fermented by lactobacillus plantarum b12 in diet-induced obese mice. J. Med. Food 2016, 19, 823-829.

[19] Kimura, S.; Tung, Y.C.; Pan, M.H.; Su, N.W.; Lai, Y.J.; Cheng, K.C. Black garlic: A critical review of its production, bioactivity, and application. J. Food Drug Anal. 2017, 25, 62-70.

[20] Bradley, J.M.; Organ, C.L.; Lefer, D.J. Garlic-derived organic polysulfides and myocardial protection. J. Nutr 2016, 146, 403S-409S.

[21] Y. Pranoto, V. M. Salokhe, and S. K. Rakshit, "Physical and antibacterial properties of alginatebased edible film incorporated with garlic oil," Food Research International, vol. 38, no.3, pp. 267-272, 2005.

[22] G.P.Sivam, "Protection against Helicobacter pylori and Other Bacterial Infections by 
Garlic,"Journal of Nutrition, vol.131, no. 3, pp. 1106S-1108S, 2001.

[23] P. Z. Trio, S.You, X. He, J. He, K. Sakao, and D.-X. Hou, "Chemopreventive functions and molecular mechanisms of garlic organosulfur compounds," Food \& Function, vol.5, no.5, pp. 833844, 2014.

[24] R. Arreola, S. Quintero-Fabian, R. I. Lopez-Roa et al., "Immunomodulation and anti-infammatory effects of garlic compounds," Journal of Immunology Research, vol.2015, Article ID 401630, p.10,2015.

[25] M. Ichikawa, J. Yoshida, N. Ide, T. Sasaoka, H. Yamaguchi, and K. Ono, "Tetrahydro-carboline derivatives in aged garlic extract show antioxidant properties," Journal of Nutrition, vol. 136, no. 3, supplement, pp. 726s-731s, 2006.

[26] C. Borek, "Antioxidant Health Effects of Aged Garlic Extract," Journal of Nutrition, vol. 131, no. 3, pp. 1010S-1015S, 2001.

[27] J. Z. Liu, X. Y. Lin, and J. A. Milner, "Dietary garlic powder increases glutathione content and glutathione S-transferase activity in rat liver and mammary
tissues,’Te FASEB Journal, vol.6, article, A3230, 1992.

[28] C. Borek, "Antioxidants and cancer,"Science \& Medicine, vol.4, pp.51-62, 1997.

[29] S. G. Santhosha, P. Jamuna, and S. N. Prabhavathi, "Bioactive components of garlic and their physiological role in health maintenance: A review," Food Bioscience, vol.3, pp. 59-74, 2013.

[30] L. Y. Chung, "Te antioxidant properties of garlic compounds: Alyl cysteine, alliin, allicin, and allyl disulfde,"Journal of Medicinal Food, vol.9, no.2, pp.205-213, 2006.

[31] N. Ide and B. H. S. Lau, "Garlic compounds minimize intracellular oxidative stress and inhibit nuclear factor-B activation," Journal of Nutrition, vol. 131, no. 3, pp. 1020S-1026S, 2001.

[32] J.-H. Park, Y.K. Park, and E.Park, "Antioxidative and antigenotoxic effects of garlic (Allium sativum L.) prepared by different processing methods,"Plant Foods for Human Nutrition,vol.64, no. 4, pp. 244-249, 2009.

[33] H.Y. Kay, J.W. Yang, T.H. Kimetal., "Ajoene, astablegarlic 
by-product, has an antioxidant effect through Nrf2 - mediated glutamate-cysteine ligase induction in HepG2 cells and primary hepatocytes," Journal of Nutrition, vol. 140, no.7, pp.1211-1219, 2010.

[34] B. H. S. Lau, "Detoxifying, radioprotective and phagocyte enhancing effects of garlic," International Clinical Nutrition Review, vol.9, pp.27-31, 1989.

[35] M. Corzomartinez, N. Corzo, and M. Villamiel, "Biological properties of onions and garlic," Trends in Food Science \& Technology, vol.18, no.12, pp. 609-625, 2007.

[36] G. Kuttan, "Immunomodulatory efect of some naturally occuring sulphur-containing compounds," Journal of Ethnopharmacology, Vol. 72, no.1-2, pp.93-99, 2000.

[37] H. Ishikawa, T. Saeki, T. Otani et al., "Aged garlic extractprevents a decline of NK cell number and activity in patients with advanced cancer, "The Journal of Nutrition, vol. 136, pp. 816S-820S, 2006.

[38] N. Morioka, L. L. Szel, D. L. Morton, and R. F. Irie, "A proteinfraction from aged garlic extract enhances cytotoxicity and proliferation of human lymphocytes mediated by interleukin-2 and concanavalin A," Cancer Immunology, Immunotherapy, vol.37, pp. 316-322, 1993.

[39] B. H. Lau, T. Yamasaki, and D. S. Gridley, "Garlic compounds modulate macrophage and $\mathrm{T}$ lymphocyte functions," Molecular Biotherapy, vol.3, pp. 103-107, 1991.

[40] M. Colic, D. Vucevic, V. Kilibarda, N. Radicevic, and M. Savic, "Modulatory effects of garlic extracts on proliferation of T-lymphocytes in vitro stimulated with concanavalin A," Phytomedicine, vol.9, no. 2,pp. 117124, 2002. 